\title{
Bases de la distribución del valor económico generado para la sustentabilidad empresarial $^{1}$
}

\author{
Basis of generated economic value \\ distribution for business sustainability
}

\author{
Karime Chahuán-Jiménez², Jonathan Vásquez Verdugo ${ }^{3}$
}

\begin{abstract}
RESUMEN
La Responsabilidad Social Empresarial (RSE) ha sido discutida durante las últimas cuatro décadas. Las demandas sociales mundiales exigen que las organizaciones reporten su impacto ambiental y social, los cuales pueden ser realizados en base a los estándares del Global Reporting Initiative (Gri). Estos guían a las empresas para mostrar el valor económico distribuido en al menos 5 aspectos, los cuales son costos operacionales, salarios y beneficios a empleados, pago a proveedores (suministros y capital), pagos al gobierno e inversiones en la comunidad. Dada esta facilidad, desde una perspectiva de parte interesada, surge la pregunta de la posible existencia en distintos focos de este valor distribuido. Luego de un análisis cualitativo exploratorio y la posterior aplicación de algoritmos de clustering ensamblados, se identificaron tres grupos en el año 2018 derivados de las empresas bajo estudio, que corresponden a las que tienen una mayor presencia en el mercado a través de sus cotizaciones bursátiles, y cinco grupos en el año 2019. Cada grupo tiene un enfoque en 4 de los 5 aspectos enlistados previamente por el GRI. De acuerdo con los resultados obtenidos, la generación de grupos permite al inversionista conocer el foco de la distribución de la generación de valor en la empresa en la cuál realizará la inversión. Adicionalmente, el concepto inversiones en la comunidad no fue identificado como característico en los grupos identificados, pudiendo existir oportunidades de mejora por parte de las empresas.
\end{abstract}

Palabras clave: clustering ensamblado, GRI, sustentabilidad y valor económico.

Recepción: 19/08/2020, Aprobación: 03/12/2020.

1 Artículo premiado en la XXXI Conferencia Anual de la Conferencia Académica Permanente de Investigación Contable.

2 Universidad de Valparaíso, Facultad de Ciencias Económicas y Administrativas, Valparaíso, Chile, karime.chahuan@uv.cl 3 Universidad de Valparaíso, Facultad de Ciencias Económicas y Administrativas, Valparaíso, Chile, jonathan.vasquez@uv.cl 


\begin{abstract}
Corporate Social Responsibility (CSR) has been discussed over the last four decades. Global social demands require organizations to report their environmental and social impact, which can be done based on the standards of Global Reporting Initiative (GRI). These establish that economic value can be distributed in at least 5 aspects: Operational Costs, Salary and Employee Benefits, Payments to Suppliers, Payments to Government, and Investments in the Community. Given this, from a stakeholder's perspective, inquiries arise about concentration in the distributed value. After an exploratory qualitative analysis followed by an Ensembled Clustering algorithm implementation, 3 and 5 groups were identified in 2018 and 2019 respectively from the companies with greater presences in the stock market. Each group has concentrations in 4 of the 5 aspects previously listed by the GRI. According to the results, the identification of these groups would allow investors to know the spotlights on the distributed value generation so using this as inputs when investments are made. Additionally, according to the results, the characteristics related to the Investments in the Community was identified as not considered by any groups, opening a gap of differentiating aspect and improvement by companies.
\end{abstract}

Key words: assembled clustering, economic value, GRI and sustainability.

\title{
INTRODUCCIÓN
}

De acuerdo con Yang (2017) la Responsabilidad Social Empresarial (RSE) ha sido ampliamente discutida durante las últimas cuatro décadas (Lu y Liu, 2014). Bowen (1953) definió a la responsabilidad social como las: "políticas, decisiones y acciones que se alinean con los objetivos y valores de la sociedad" Yang (2017, 1494). Por otro lado, Davis (1960) definió la: "RSE como las acciones y decisiones que toman los profesionales del área de negocios por motivos que no sean económicos o técnicos" mientras que Becchetti y Trovato (2011) afirmaron que la: "RSE implica un cambio de enfoque de la maximización de la riqueza de los accionistas a la de una función de bienestar de múltiples partes interesadas" Yang (2017: 1494), y recientemente, Kang y Liu (2014) señalaron que la RSE es un término complejo que implica la contribución activa y / o voluntaria de los recursos empresariales a acciones destinadas a lograr mejoras ambientales, sociales y económica.

La demanda social mundial exige que las organizaciones sean responsables de su impacto ambiental y social (Brown y Dillard, 2014; Park y Brorson, 2005; Rasche y Esser, 2006, citado por Safiri y Areeb (2020)). Para responder a estas presiones y comunicar el desempeño de la RSE a las partes interesadas, un número creciente de organizaciones publica informes de sostenibilidad (KPMG (2015) citado por Safiri y Areeb (2020) y Smit y Bierman (2017)). Estos informes son ampliamente utilizados por las partes interesadas y se considera que poseen un gran impacto en el estado corporativo, así como en las ventajas competitivas (Boiral 2013; Boiral, Heras-Saizarbitoria y Brotherton, 2019; Schultz y Wehmeier, 2010, citado en Safiri y Areeb (2020)). En la literatura se ha argumentado que la utilización del marco de Global Reporting Initiative (GRI) mejora la estandarización de los informes de sostenibilidad (Moneva, Archel y Correa, 2006). Sin embargo, a pesar de los esfuerzos de GRI por desarrollar un marco que ayude a las organizaciones a mejorar su verdadero impacto en la sostenibilidad, estudios muestran que los informes de sostenibilidad 
a menudo se utilizan como herramientas para la legitimación social (Campbell, 2003; Deegan, 2002, 2007; Hahn \& Lulfs, 2014; Manetti, 2011, Safiri y Areeb, 2020).

De acuerdo con el GRI (2016), la información sobre la creación y la distribución del valor económico indica de forma básica cómo una organización ha generado riqueza a los grupos de interés. La recopilación de información de su desempeño económico utiliza cifras que se recogen en sus estados financieros auditados o en sus cuentas de gestión auditadas internamente, siempre que sea posible. Es posible recopilar datos utilizando, por ejemplo: "Los estándares internacionales para la elaboración de informes financieros (IFRS), publicados por la Junta de Normas Internacionales de Contabilidad (IASB), y las interpretaciones del Comité de Interpretaciones de los IFRS (se hace referencia a algunos IFRS concretos para ciertas informaciones); los estándares internacionales de contabilidad para el sector público (IPSASB), y los Estándares nacionales o regionales reconocidos a nivel internacional para la elaboración de informes financieros" (GRI, 2016, 6).

En el caso de las empresas chilenas, estas han avanzado en la presentación de informes sustentables, reportes sustentables o memorias integradas, con base en el GRI, exponiendo la incorporación de cada ítem en un apartado informativo en sus informes financieros o a través de sus reportes sustentables o integrados. Desde el área económica presentan sus valores económicos generados y distribuidos, variando este último entre empresas. Desde una perspectiva de las partes interesadas, surge la pregunta de la existencia de focalización sobre la distribución del valor económico que tienen las empresas chilenas en función de la información entregada a través de los reportes sustentables y /o integrados de los años 2018 y 2019. En este sentido, el objetivo de la investigación es exponer los focos que existen en las empresas que reportan la información en base a la conceptualización entregada por el GRI sobre la distribución del valor económico generado por las empresas.

La investigación se basa en un enfoque mixto (Creswell y Creswell, 2017), en específico, en el exploratorio secuencial, cuya primera etapa es cualitativa y la segunda cuantitativa. Primero, a través de un análisis documental de los reportes de sustentabilidad, se identifican y categorizan la distribución que realizan las empresas en los conceptos entregados por el GRI, a decir, en costos operacionales, salarios y beneficios de los empleados, pagos a proveedores de capital, pagos al gobierno (por país), e inversiones en la comunidad. Posteriormente, en una segunda etapa cuantitativa, se aplican técnicas de clustering para identificar grupos de empresas con distribución del valor económico similar tanto para el año 2018 y 2019. Este permitiría identificar el enfoque existente sobre la distribución del valor agregado, así como también la variación intra e intergrupal entre ambos años, basándose en los cambios que reportaron las empresas en su distribución del valor. Este novedoso enfoque permite identificar la existencia de focos que las empresas reportan respecto a su inversión en la generación de valor, permitiendo a inversionistas, financistas e interesados (stakeholders) obtener una nueva perspectiva de información para la toma de decisiones. De acuerdo con el conocimiento de los autores de la literatura, no existe un enfoque similar de identificación de grupos y categorías en lo que respecta a la distribución de valor agregado.

Este articulo está compuesto por una sección de metodología, material y métodos, en el que se detalla las etapas establecidas para la recopilación de los datos a utilizar. Posteriormente está la sección de análisis de tendencias por empresa y sector según los datos recopilados; en esta 
sección se discutirán también los hallazgos obtenidos. Finalmente, la última sección trata las conclusiones, limitaciones y proyecciones generadas de la investigación.

\section{METODOLOGÍA, MATERIAL Y MÉTODOS}

Las empresas analizadas forman parte del IPSA (Índice de Precio Selectivo de Acciones), correspondiente a las 29 firmas con una mayor cantidad de transacciones bursátiles para el año 2018-2019 y se consideran los antecedentes de sus memorias anuales e informes de sustentabilidad publicados. Los principales rubros de la muestra son: Generación y distribución de suministro de agua y electricidad, minería, intermediación financiera, forestal, combustible, retail, embotelladoras, comunicaciones, vitivinícola, tecnológicas, y de inversión. A continuación, en la tabla 1 se presenta una caracterización de las empresas que componen el IPSA con datos del 2018.

Tabla 1

Caracterización de las empresas del IPSA con datos del año 2018.

\begin{tabular}{|l|r|r|r|r|r|r|}
\hline & $\mathrm{N}$ & \multicolumn{1}{c|}{ Rango } & \multicolumn{1}{c|}{ Mínimo } & \multicolumn{1}{c|}{ Máximo } & \multicolumn{1}{c|}{ Media } & Desv. Error \\
\hline $\begin{array}{l}\text { Ganancia Neta } \\
\text { (M CLP) }\end{array}$ & 29 & 859.402 & -23.614. & 835.788 & 240.252 & 42.049 \\
\hline $\begin{array}{l}\text { Ingresos Netos } \\
\text { (M CLP) }\end{array}$ & 29 & 16.612 .126 & 63.611 & 16.675 .737 & 2.816 .123 & 693.151 \\
\hline $\begin{array}{l}\text { Liquidez } \\
\text { (Índice: } \frac{\text { Activo corriente }}{\text { Pasivo corriente }} \text { ) }\end{array}$ & 29 & 9,74 & 0,48 & 10,22 & 3,3428 & 0,48479 \\
\hline $\begin{array}{l}\text { Utilidad por Acción } \\
\text { (Índice: } \frac{\text { Utilidad Anual }}{\text { NASP }} \\
\text { donde NASP = Num } \\
\text { de Acciones Suscriptas } \\
\text { y Pagadas) }\end{array}$ & 29 & 3.046 & -78 & 2.967 & 286 & 113 \\
\hline
\end{tabular}

Nota: para efectos de la caracterización se han calculado los valores promedio de las empresas que componen el Índice de Precios Selectivo de Acciones. Fuente: Elaboración propia en base a datos Bolsa de Santiago (2020).

La tabla 1 contiene los estadísticos de tendencia central y variación que permiten caracterizar la muestra respecto a la acción de las empresas para el 2018, puesto que fue el año base para la selección de las empresas pertenecientes al IPSA. Para objetivos de comparabilidad, la misma lista fue usada para el análisis del año 2019. Es importante destacar que la media de las ganancias entre las empresas bajo estudio es de 240.252 .737 millones de pesos, y los ingresos corresponden a 2.816.123.069 millones de pesos. Estos parámetros son una de las bases que se ha considerado para la distribución de este valor generado.

La primera etapa consistió en la captura de los datos distribuidos por medio de un enfoque cualitativo con alcance exploratorio. Los datos primarios utilizados fueron los reportes emitidos 
por las empresas bajo estudio, de los cuales se consideraron las variables presentadas por el GRI en su ítem 201 respecto de la distribución del valor económico.

Los conceptos asociados a la información incorporada en el valor económico distribuido, se presenta una definición operacional que permite entender el análisis en base a las definiciones expuestas por GRI (2016), los que son presentados en Cuadro 1.

\section{Cuadro 1}

Definición operación de los conceptos involucrados en el valor económico distribuido.

\begin{tabular}{|l|l|}
\hline \multicolumn{1}{|c|}{ Concepto } & \multicolumn{1}{c|}{ Definición operacional } \\
\hline Costos operacionales. & $\begin{array}{l}\text { Las organizaciones pueden calcular los costos operacionales tenien- } \\
\text { do en cuenta los pagos en efectivo -realizados fuera de la organi- } \\
\text { zación- de materiales, componentes de productos, instalaciones y } \\
\text { servicios adquiridos. (GRI, 2016: 7) }\end{array}$ \\
\hline $\begin{array}{l}\text { Salarios y beneficios a } \\
\text { los empleados. }\end{array}$ & $\begin{array}{l}\text { Las organizaciones pueden calcular los salarios y beneficios de los } \\
\text { empleados sumando las nóminas totales (incluidos los salarios de } \\
\text { los empleados y las cantidades pagadas a instituciones gubernamen- } \\
\text { tales en nombre de los empleados) y los beneficios (excluidos la for- } \\
\text { mación, los costes de los equipos de protección y los costes de artícu- } \\
\text { los directamente relacionados con la función laboral del empleado). } \\
\text { (GRI, 2016: 7) }\end{array}$ \\
\hline $\begin{array}{l}\text { Pago a proveedores de } \\
\text { capital y suministros. }\end{array}$ & $\begin{array}{l}\text { Las organizaciones pueden calcular los pagos a proveedores de ca- } \\
\text { pital sumando los dividendos de todos los accionistas y los pagos de } \\
\text { intereses realizados a los distintos prestamistas. (GRI, 2016: 7) }\end{array}$ \\
\hline Pagos al gobierno. & $\begin{array}{l}\text { Las organizaciones pueden calcular los pagos a los gobiernos su- } \\
\text { mando todos los impuestos de la organización y las sanciones rela- } \\
\text { cionadas pagadas a nivel internacional, nacional y local. Los impues- } \\
\text { tos de la organización pueden incluir los impuestos de sociedad, las } \\
\text { ganancias y la propiedad inmobiliaria. (GRI, 2016: 7) }\end{array}$ \\
\hline $\begin{array}{l}\text { Inversiones en la comu- } \\
\text { nidad }\end{array}$ & $\begin{array}{l}\text { Las inversiones totales en la comunidad hacen referencia a los gastos } \\
\text { reales efectuados durante el periodo objeto del informe y no a los } \\
\text { compromisos. Las organizaciones pueden calcular las inversiones en } \\
\text { la comunidad sumando las donaciones voluntarias y la inversión de } \\
\text { fondos en la comunidad más amplia cuyos beneficiarios no formen } \\
\text { parte de la organización. (GRI, 2016: 7) }\end{array}$ \\
\hline
\end{tabular}

Nota: La definición operacional para las categorías de análisis son las que presenta el Global Reporting Initiative, cuando presenta su sección 201. Fuente: Elaboración propia en base a GRI (2016).

Los reportes obtenidos, en especial la información presentada respecto a la distribución del valor económico generado, se revisaron y categorizó a través del software ATLAS.ti. Este análisis se llevó a cabo en todas las empresas de la muestra, en donde se codificó la sección correspondiente a la información buscada. Una vez identificada la información en cada uno de los reportes, 
se consolidaron los datos necesarios en una plantilla para ser analizada por medio de un algoritmo de clusterización en la siguiente etapa.

Tal como se mencionó, una vez categorizados los conceptos de valor económico distribuidos, se estructuraron los datos en tablas de recopilación en planillas excel, las que permitirían la realización del análisis e identificación de grupos similares a través del algoritmo de clustering.

Los datos obtenidos fueron utilizados para identificar grupos similares de empresas de acuerdo con criterios de similitud. Para esta tarea se utilizó la técnica de Clustering Ensamblado (Alqurashi \& Wang, 2019; Fred \& Jain, 2005), el que inicialmente genera un conjunto de agrupaciones, conocidas como particiones, con distintos enfoques de clusterización. Luego, por medio de una función de consenso, se identifica una agrupación final, la que considera todas las particiones generadas previamente. Esto resultaría en una mejor solución en comparación de realizar una partición con un solo algoritmo, el cual es el enfoque regular de clustering.

En el procedimiento inicial, las particiones se obtienen aplicando distintos algoritmos de clustering, tales como el k-means, fuzzy c-means, y HDBSCAN (Arora \& Varshney, 2016; Bezdek et al., 1984; McInnes et al., 2017). Posteriormente, se evalúa la similitud entre todas las observaciones (para los efectos de la investigación, las empresas) tomando en cuenta las particiones generadas en la primera etapa y usando mediciones de función de consenso. Un ejemplo de medición de consenso es la coocurrencia que calcula la similitud entre dos observaciones (empresas), contando la coocurrencia de ser asignadas al mismo grupo en las particiones de la primera etapa. Posteriormente se obtiene una agrupación final considerando la similitud obtenida según función y un algoritmo que utilice esta medida para volver a agrupar las observaciones.

Uno de los más comunes son los métodos de clusterización jerárquicos, los cuales generan grupos partiendo con una separación simple de dos grupos hasta ir segregándolos y llegar finalmente a una agrupación final (Provost \& Fawcett, 2013). También se usan otros algoritmos más convencionales, tales como el Majority Voting, un sistema de voto que designa cada registro a aquel grupo que fue mayoritariamente asignado entre todas las particiones. Por su diseño, los algoritmos basados en agrupaciones jerárquicas entregan más de una opción de números de grupos. Esto implica que se debe identificar el número óptimo de grupos necesarios por medio de indicadores de desempeños, tales como las distancias intragrupal o intergrupal. También existen mediciones más robustas, tales como la porción de ambigüedad de los clusters (PAC), el cual identifica el grado de ambigüedad en los grupos (Șenbabaoğlu et. al, 2014).

En esta investigación fue implementado el algoritmo previamente descrito en RStudio y se utilizó el paquete de librería diceR del repositorio CRAN (Chiu \& Talhouk, 2018; diceR package I $\mathrm{R}$ Documentation, 2019). Además, para la implementación se usaron los algoritmos de partición $k$-means, fuzzy c-means, y HDBSCAN, evaluando particiones en diferentes números de grupos $(k=\{2,3,4,5,6\})$ y con un número de iteraciones $t=100$. Los resultados de cada uno fueron utilizados para calcular la similitud entre las observaciones por medio de los algoritmos de consenso Majority Voting y K-modes. Con los datos obtenidos de las empresas en la etapa I se aplicaron dos clusteriaciones, uno para el año 2018 y otro para el 2019, obteniendo así dos resultados de agrupaciones, los cuales son discutidos en la siguiente sección. 


\section{RESULTADOS}

La categorización de la información permitió agrupar a las empresas que entregan información asociada a la distribución del valor económico distribuido, considerando a las empresas con mayor nivel de transacciones. Esto, puesto que hay varios factores que indican que las empresas deben cambiar su formato por expectativas de los inversores y se ven afectadas por las decisiones de inversiones del mercado. Considerando las empresas bajo estudio, se pudo identificar que hay empresas cuya información reportada no se presenta en los formatos y en la organización que expone el GRI, dificultando la facilidad de identificar la información necesaria puesto que se encuentra distribuida a lo largo del reporte sustentable. Por otro lado, hay aspectos que no se informan en valores monetarios, sino que se describen desde un punto de vista cualitativo. Esto dificulta el análisis cuantitativo, tal como el realizado en este estudio.

Para facilitar la interpretación de los resultados obtenidos por el Clustering Ensamblado, se generan gráficas que permiten analizar visualmente las agrupaciones finales. Para esto, se utiliza las librerías caret y fmsb, ambas almacenadas en el repositorio CRAN de RStudio. La primera figura es un gráfico de dispersión, en donde se generan dos dimensiones por medio del análisis PCA (Principal Component Analysis), el cual identifica dimensiones que son combinaciones lineales de las características de los datos. El PCA identifica aquellas nuevas dimensiones que maximizan la variabilidad de los datos, por lo que un porcentaje alto en una dimensión generada significaría que esta refleja en gran medida la variabilidad de los datos. En otras palabras, se grafican las 5 características obtenidas de cada empresa en 2 dimensiones. En los resultados, las dos dimensiones identificadas por el PCA, y que son utilizadas para graficar, reflejan en un $61,7 \%$ y $63,5 \%$ la variabilidad de los años 2018 y 2019 respectivamente.

Finalmente, se generan dos nuevos gráficos presentados a través de las figuras apropiadas con el objetivo de comparar los perfiles de cada grupo. El primero es del tipo telaraña y se utiliza en los resultados del año 2018. En él se muestra el valor representativo de cada grupo en las 5 características. Por otro lado, el segundo gráfico presentado en la Figura 3 es de coordenadas paralelas y se utilizan los resultados del año 2019. En este tipo de gráfico se ordena de izquierda a derecha las dimensiones en ejes paralelos y se traza una línea por cada observación. En ambas figuras se utilizan los centroides de cada grupo, el cual es una observación representativa de los grupos, cuyos valores se obtienen calculando el valor promedio en cada una de las 5 características.

Para el año 2018 se identifican 3 grupos. Hay que notar que al centro de cada grupo se muestra el centroide, siendo un círculo para el grupo 1, un triángulo para el grupo 2, y un cuadrado para el grupo 3. De acuerdo con la Figura 1, visualmente se observa que el grupo 3 tiene mayor similitud intragrupal, mientras que el grupo 1 es el que refleja menos similitud entre los grupos. Por otro lado, existe una diferencia clara entre estos tres grupos, los cuales se analizan más en detalle en el Figura 2. Adicionalmente, y complementando con los análisis cualitativos de la Etapa 1 de la metodología, se puede indicar que el grupo 3 se compone principalmente por aquellas compañías que mejor reportan el valor económico distribuido según GRI. 


\section{Figura 1}

Representación gráfica de los grupos obtenidos utilizando PCA, año 2018.

\section{Cluster 2018}

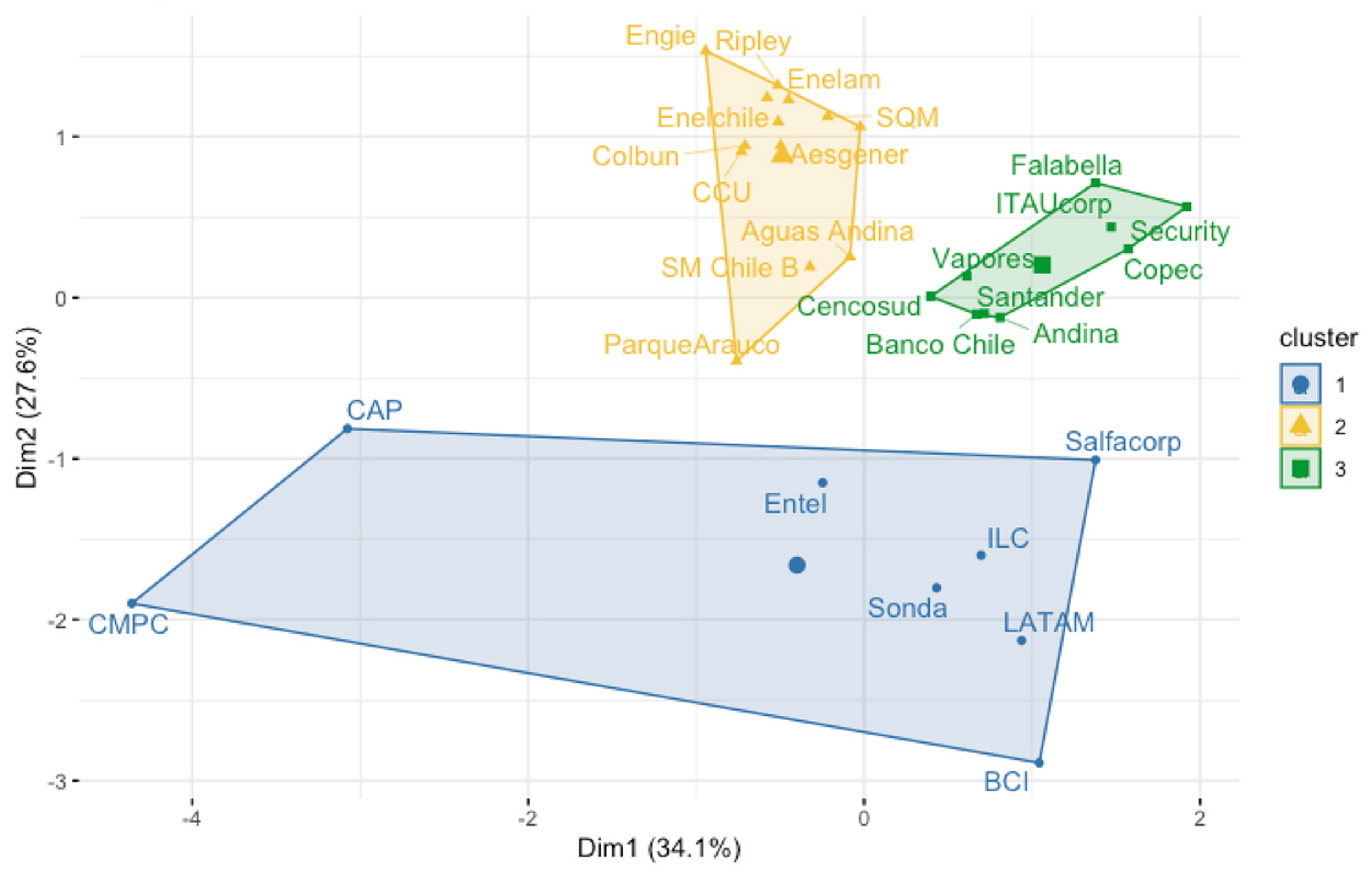

Fuente: Elaboración propia.

Según Figura 2, los grupos se diferencian principalmente por tres características: (1) el Costo Operacional, (2) el Salario y Beneficio a Empleados, y (3) el Pago a Proveedores. Se observa claramente que el grupo 2 destaca en el grado de Costo Operacional reportado, el grupo 1 en lo que respecta a Salario y Beneficio a Empleados, y finalmente, el grupo 3 en el Pago a Proveedores. En términos de Inversiones en la Comunidad y Pago Gobierno, los tres grupos son bastantes similares, en donde se reportan niveles bajos. 


\section{Figura 2}

Perfiles de los grupos en gráfico de telaraña basado en los centroides, año 2018.

\section{8}

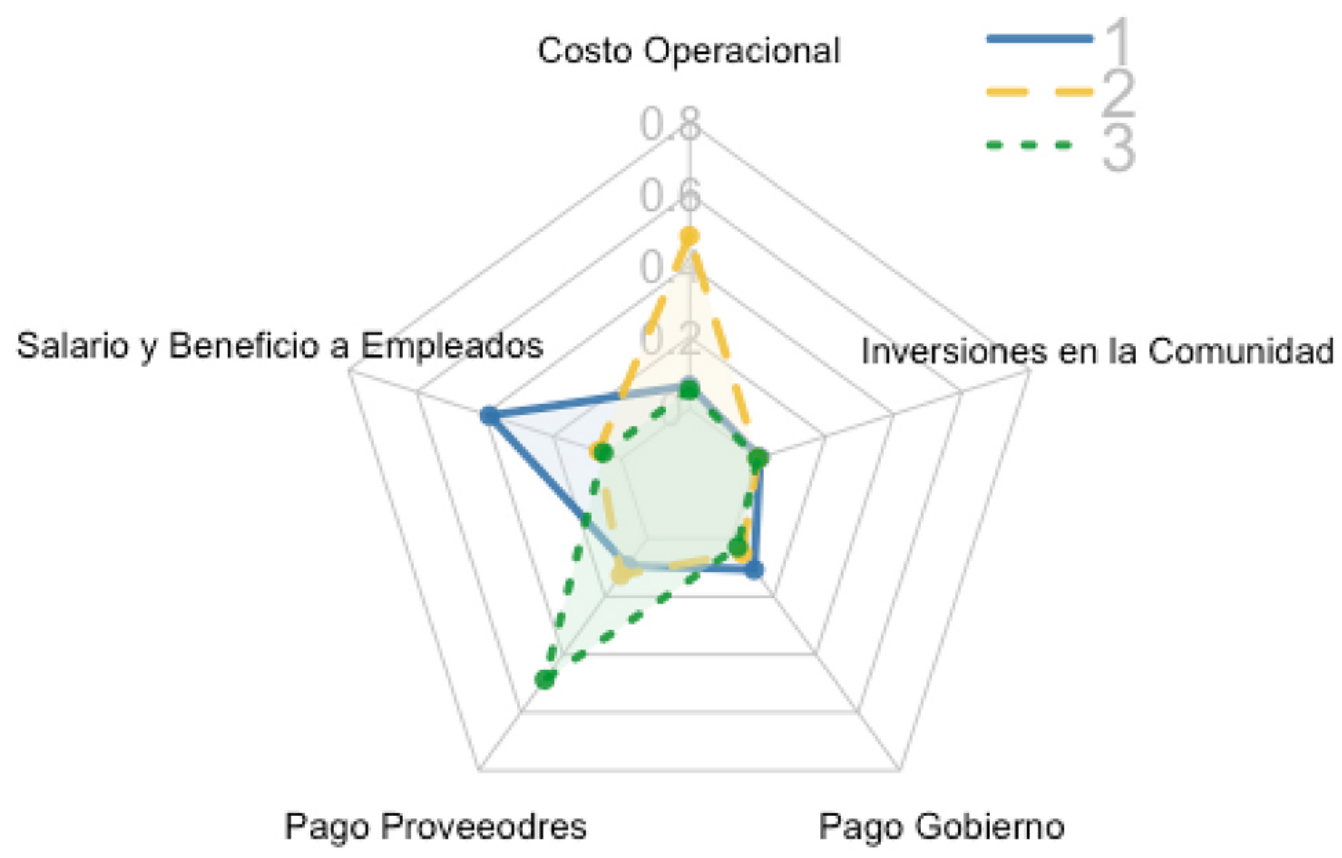

Fuente: Elaboración propia.

\section{Año 2019}

Para el año 2019 se identifican dos nuevos grupos; uno relacionado con la separación de la compañía CMPC con su grupo del año 2018 y otro nuevo compuesto por Cencosud, Itaú, Copec, CAP, Falabella y Security. Esto se observa porque para el 2019 la empresa CAP, que estaba cercana a CMPC, ahora se asimila más con las empresas que componían el grupo de Cencosud, Itau y Copec en el año 2018. Por otro lado, el grupo 2 casi no sufre variación respecto del año anterior, y el grupo 3 del año 2018, considerado como el que mejor reporta, crece en tamaño. Cabe destacar que los grupos 3 y los dos nuevos no tienen tanta distancia entre ellos, lo que se muestra en la Figura 3. 


\section{Figura 3}

Representación gráfica de los grupos obtenidos utilizando PCA, año 2019.

\section{Cluster 2019}

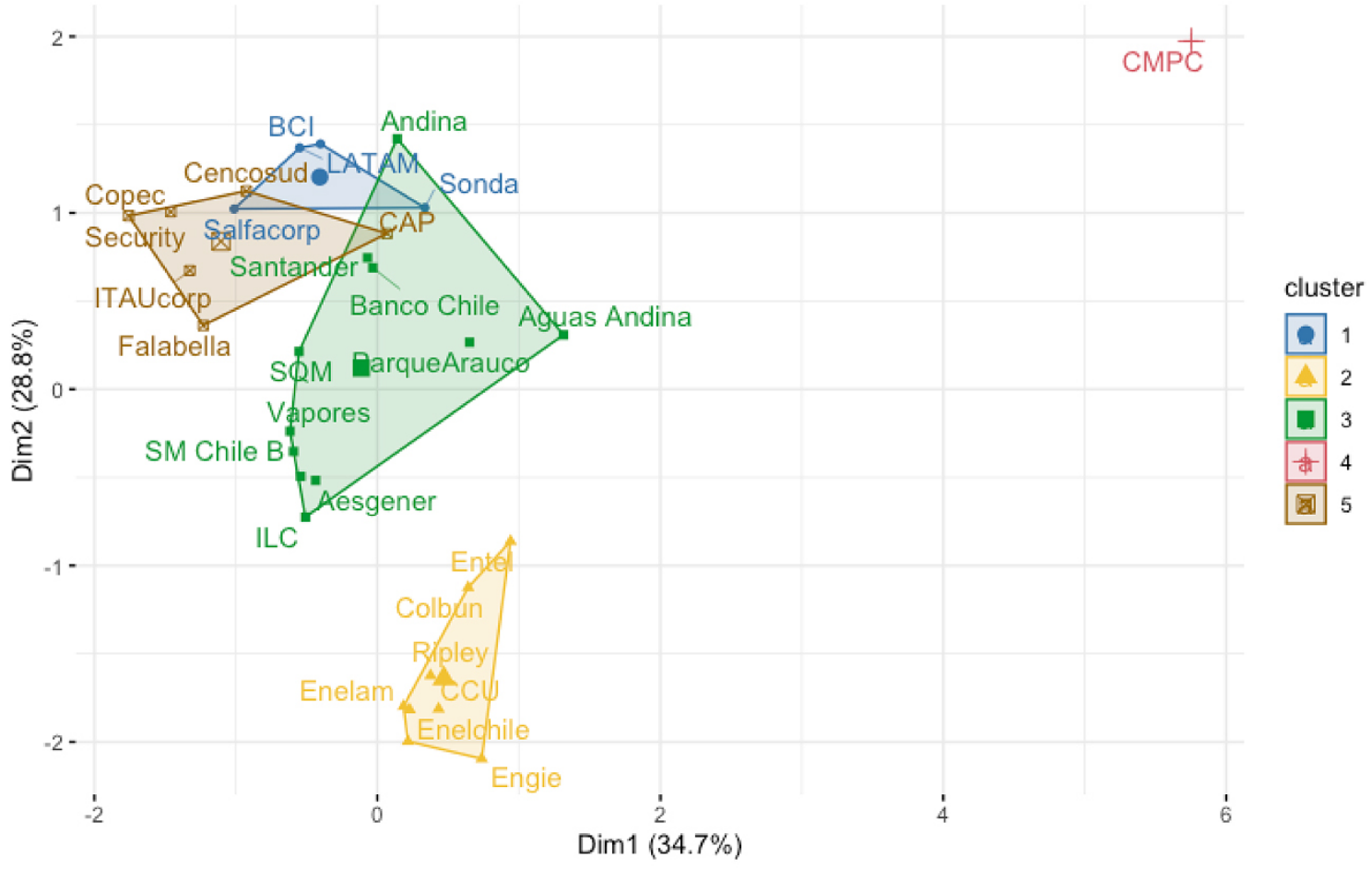

Fuente: Elaboración propia.

La Figura 4 de perfiles muestra los perfiles por medio de coordenadas paralelas; esta decisión fue tomada por conveniencia visual. Según la figura, se mantienen las diferencias reflejada para los grupos 1, 2 y 3 del año 2018. Además, los nuevos grupos tienen ciertas similitudes con los identificados en el año 2018. En otras palabras, el grupo 4 se asimila con el grupo 1 y 2, pero adicionalmente es el que más reporta respecto a la característica Pago Gobierno. Hay que recordar que este nuevo grupo está compuesto solamente por CMPC. Por otro lado, el grupo 5 pareciese ser una separación del grupo 3 y con algunas características del grupo 2 en lo que respecta a Costo Operacional. 


\section{Figura 4}

Perfiles de los grupos en gráfico de coordenadas paralelas basado en los centroides, año 2019.

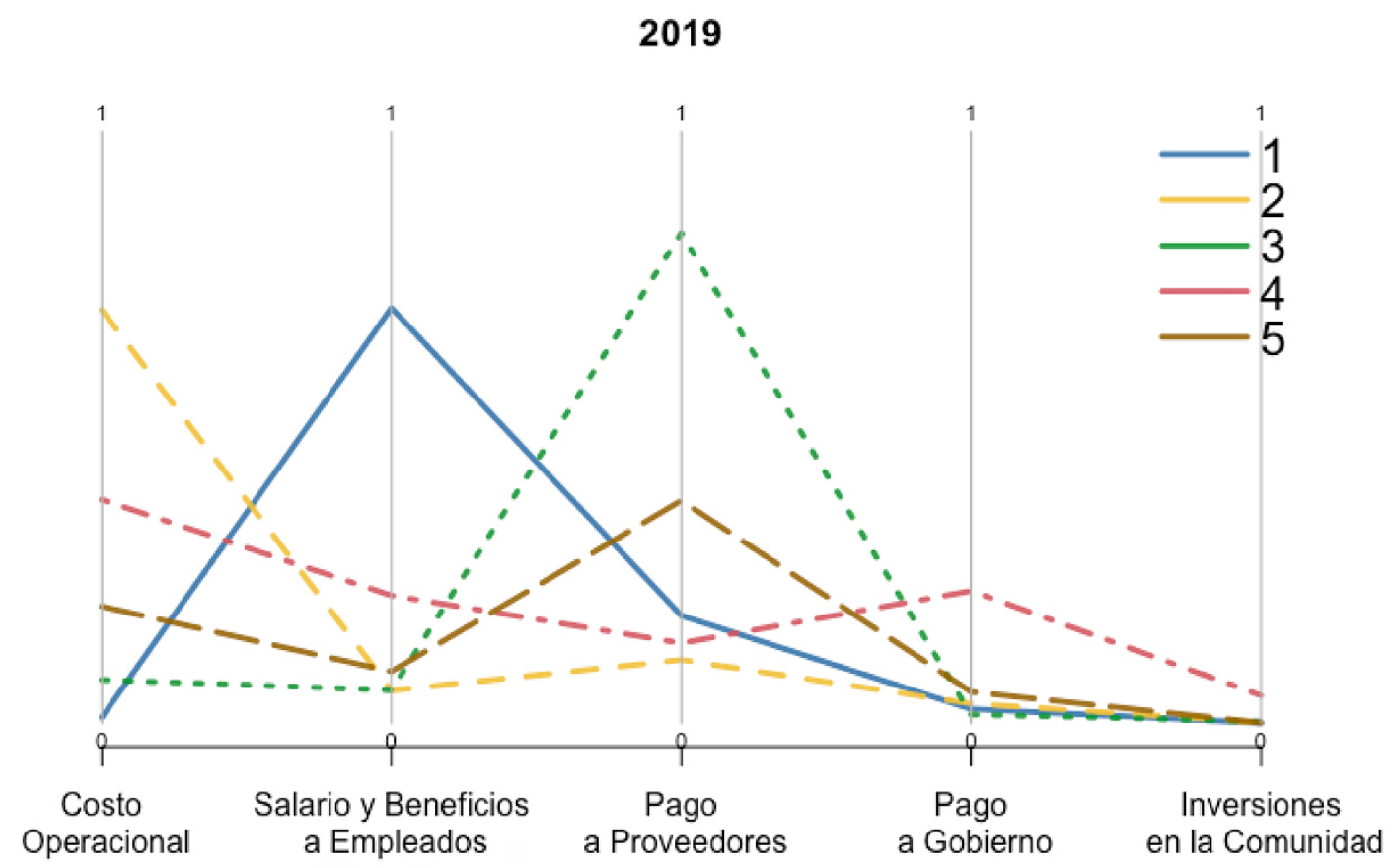

Fuente: Elaboración propia.

De acuerdo con las agrupaciones considerando las variables que componen la distribución del valor económico generado en ambos años, es posible identificar los 3 y 5 grupos para el año 2018 y 2019 respectivamente. En ambos años se observan marcadamente 3 focalizaciones, relacionados con las dimensiones de Costo Operacional, Salario y Beneficios a Empleados, y Pago a Proveedores del valor económico distribuido. Adicionalmente, para el año 2019 se identifican dos nuevos grupos, siendo uno de ellos muy parecido al grupo con foco en Pago a Proveedores y otro que se diferencia con un leve enfoque en Pago a Gobierno. Cabe destacar que en ambos años el dominio Inversiones en la Comunidad es bajo, pudiendo identificarse un espacio de diferenciación por parte de las empresas.

Este análisis basado en clustering agregar un elemento adicional informativo a los inversionistas, pues, desde el punto de vista sustentable su decisión de inversión puede estar basada en función de criterios de la distribución de valor generado por las compañías, la cual se obtiene de información pública, expresada en montos en pesos y dólares por las empresas. 


\section{DISCUSIÓN Y CONCLUSIONES}

Según Safiri y Areeb (2020), la literatura ha mostrado que los informes de sustentabilidad a menudo se utilizan como herramientas para la legitimación social desde las distintas partes interesadas en las empresas. Razón por ello el GRI presenta un ordenamiento base para reportar el valor económico distribuido, permitiendo entregar transparencia de la información, vinculándolo con las políticas, decisiones y acciones que se alinean con los objetivos y valores de la sociedad de acuerdo con Yang $(2017,1494)$. Acorde con los procesos metodológicos desarrollados basados en los informes sostenibles los que de acuerdo con Safiri y Areeb (2020) estos son ampliamente utilizados por las partes interesadas y generan una ventaja competitiva para las empresas que lo presentan, es posible verificar que hay empresas que no exponen en sus reportes las categorías valorizadas propuestas por el GRI, adicionalmente la información que indica que se presentara de manera pública la forma básica en como una organización ha generado riqueza los grupos de interés (GRI, 2016). Además, entregan una mayor cantidad de información cualitativa, lo que no permite que los datos se hagan comparables desde una perspectiva cuantitativa. Para lograr este último punto, es necesario aplicar un proceso exploratorio previo, tal como el propuesto e implementado en esta investigación.

Considerando los grupos generados a través de Clustering Ensamblado, considerando de acuerdo con Yang (2017), que las inversiones de las empresas en RSE implica un bienestar a múltiples partes interesadas, en función de la distribución del valor económico generado, se pudo identificar para el año 2018 tres grupos de empresas focalizadas en Costo Operacional, Salario y Beneficios a Empleados y Pago a Proveedores respectivamente. Para el año 2019, los 3 grupos quedan en 5 grupos, manteniendo las mismas bases conceptuales del año 2018 en tres grupos, pero se crean dos nuevos. Se destaca que uno de ellos se compone por un solo integrante y se aleja levemente por la característica asociada al Pago a Gobierno.

En el caso de inversiones en la comunidad, es bastante bajo el porcentaje informado en comparación con la cantidad de actividades que desarrollan las empresas en nuestro análisis cualitativo. Esto puede reflejar dos aspectos: (1) hay un espacio de diferenciación no cubierto por la muestra analizada, ya que no están invirtiendo lo suficiente en la comunidad, como lo indica Campbell, 2003; Deegan, 2002, 2007; Hahn \& Lulfs, 2014; Manetti, 2011, Safiri y Areeb, 2020, en que se indica que los reportes se presentan como una legitimación social o (2) las empresas analizadas no están entregando toda la información en relación con su inversión en la comunidad, lo que afecta el nivel de desinformación de los inversionistas a la hora de usar esta información para la toma de decisiones, dado que en Chile las empresas están avanzando en la información pública sustentables pero el avance ha sido paulatino. Esto último punto es de suma importancia, tanto desde un punto de vista cualitativo como cuantitativo, puesto que la lucha por el desarrollo ambiental, social y de gobernanza es una demanda social mundial, en el que se exige a las organizaciones ser responsable en su actuar en la sociedad.

La metodología implementada nos permitió identificar cuál es la base o foco de distribución de resultado, tanto en una perspectiva cualitativa y cuantitativa. Al revisar las empresas que han sido incorporadas en cada grupo, se desataca el grupo 3 en que, si bien su foco es el Pago a Proveedores, el análisis cualitativo mostró que también hay una intención de un mejor reporte sustentable por la información entregada, como también la organización del reporte.

Teniendo en cuenta el desarrollo de la investigación y el enfoque metodológico desarrollado, la investigación logra mostrar las bondades entregadas por un enfoque mixto, el cual permite indagar un fenómeno 
desde perspectivas tanto inductivas y deductivas. Los resultados obtenidos son de gran importancia para la entrega de información a las diferentes partes interesadas. Los autores consideran que una mejor y mayor cantidad de información en los reportes permitiría continuar el desarrollo de la investigación en los años siguientes, de modo de identificar tendencias, realizar trazabilidad, y así complementar la información entregada a los agentes interesados, en especial a lo relacionado con lo sustentable para el logro de sus objetivos y la colaboración con el desarrollo ambiental, social y de gobernanza. Finalmente, bajo el conocimiento de estos investigadores, los métodos aplicados son novedosos y permiten obtener valiosa información para la identificación de nuevos patrones en lo que respecta a la reportabilidad según GRI.

\section{REFERENCIAS BIBLIOGRÁFICAS}

Alqurashi, T. \& Wang, W. (2019). Clustering ensemble method. International Journal of Machine Learning Cybernetics, 10(6), 1227-1246. doi:https: / / doi.org/10.1007/s13042-018-0807-8.

ATLAS.ti (2016). Software ATLAS.ti, Análisis cualitativo de datos. Extraído el 12 de Junio de 2020.

Arora, P. \& Varshney, S. (2016). Analysis of k-means and k-medoids algorithm for big data. Procedia Computer Science, 78, 507-512. doi:https:/ / doi.org/10.1016/j.procs.2016.02.095

Bezdek, J. C., Ehrlich, R., \& Full, W. (1984). FCM: The fuzzy c-means clustering algorithm. Computers $\mathcal{E}$ Geosciences, 10(2-3), 191-203.

Bolsa de Santiago (2020). SP IPSA. Disponible en: https: / / www.bolsadesantiago.com/detalle_indice/SP\%20IPSA. Extraído el 20 de Marzo de 2020.

Chiu, D.S., \& Talhouk, A. (2018). DiceR: An R package for class discovery using an ensemble driven approach. BMC Bioinformatics, 19(1), 11. https: / / doi.org/10.1186/ s12859-017-1996-y

Creswell, J.W., \& Creswell, J.D. (2017). Research design: Qualitative, quantitative, and mixed methods approaches. Sage publications.

diceR package I R Documentation. (2019). Extraido el 26 de Junio de 2020, https: / / www.rdocumentation.org/ packages/ diceR/versions/0.6.0

Fred, A.L., \& Jain, A.K. (2005). Combining multiple clusterings using evidence accumulation. IEEE transactions on pattern analysis and machine intelligence, 27(6), 835-850. doi:https: / / doi. org/10.1109/tpami.2005.113

GRI (2016). Gri 201: Desempeño Económico 2016. Disponible en: https://www.globalreporting.org / standards / gri-standards-translations / gri-standards-spanish-translations-download-center/ Extraído el 03 de Abril de 2020.

McInnes, L., Healy, J. \& Astels, S. (2017). Hdbscan: Hierarchical density based clustering. Journal of Open Source Software, 2(11), 205.

Moneva, J.M, Archel, P. Y Correa, C. (2006). GRI and the camouflaging of corporate unsustainability. Accounting Forum. 30(2). 121-137. https:/ / doi.org/10.1016/j.accfor.2006.02.001

Provost, F. \& Fawcett, T. (2013). Data Science for Business: What you need to know about data mining and data-analytic thinking. "O'Reilly Media, Inc.".

Safiri, M. \& Areeb, A. (2020): A qualitative analysis of GRI principles for defining sustainability report quality: an Australian case from the preparers' perspective, Accounting Forum, DOI: 10.1080/01559982.2020.1736759

Șenbabaoğlu, Y., Michailidis, G., \& Li, J.Z. (2014). Critical limitations of consensus clustering in class discovery. Scientific reports, $4(1), 1-13$. 
Smit, A. \& Bierman, E. (2017). An evaluation of the reporting on ethics and integrity of selected listed motor vehicle companies. African Journal of Business Ethics. Vol 11 (1) 82- 102. DOI: https: / / doi.org/10.15249/11-1-152

Yang, F.C. (2017). Integrating corporate social responsibility and profitability into best practice selection: the case of large Taiwanese firms. Quality $\mathcal{E}$ Quantity, 51(4), 1493-1512. doi:10.1007 / s11135-016-0348-8

\section{(c)}

Esta obra está bajo una licencia de Creative Commons Atribución-NoComercial-CompartirIgual 4.0 Internacional 